\section{Chylous Ascites in Nephrotic Syndrome}

A scites is a common feature in children with nephrotic syndrome, and if not treated early, it may gradually increase. The fluid is a transudate with a very low protein content and few cells. Occurrence of chylous ascitic fluid has been occasionally reported in adult patients with nephrotic syndrome, usually being caused by obstruction to lymphatics [1]. We report the case of a child with steroid resistant nephrotic syndrome (SRNS) who developed massive ascites. Paracentesis revealed the chylous nature of the fluid. Such a feature is rare and not well explained.

A 6-year-old child with SRNS was referred to us for with massive ascites, respiratory distress and oliguria. He was diagnosed 1 year back with SRNS, following no response to the standard treatment with prednisolone $(2 \mathrm{mg} / \mathrm{kg}$ daily for 6 weeks followed by $1.5 \mathrm{mg} / \mathrm{kg}$ on alternate days for 6 weeks). A renal biopsy was advised but declined by the parents seeking alternative treatment. The child developed abdominal distension about 8 months back, which had recently increased significantly to cause difficulty in breathing and decreased urine output. There was no associated history of jaundice or upper gastrointestinal bleeding or any other systemic illness.

Examination showed a severely malnourished child with massive ascites respiratory distress, facial and pedal edema, marked pallor and cold peripheries. Vitals revealed tachycardia, tachypnea and a blood pressure of $80 / 40 \mathrm{mmHg}$. On laboratory evaluation the hemoglobin was $6.2 \mathrm{~g} / \mathrm{dL}$, serum albumin $1.4 \mathrm{~g} / \mathrm{dL}$, globulin $1.4 \mathrm{gm} / \mathrm{dL}$, urea $101 \mathrm{mg} / \mathrm{dL}$ and creatinine $1.7 \mathrm{mg} / \mathrm{dL}$. The levels of serum electrolytes, bilirubin and liver enzymes were within normal range. Fasting lipid profile including serum cholesterol (138 mg/dL) was normal. Urine showed 4+ protein and no red cells on microscopy. Fluid resuscitation was done with $0.9 \%$ saline following which peripheral perfusion improved but oliguria persisted (urine output $100 \mathrm{ml}$ in first 24 hour). He was given 20\% albumin infusion and diuretics. Abdominal paracentesis was done to relieve respiratory distress. Paracentesis revealed milky white fluid, which on analysis showed protein content of $1.2 \mathrm{gm} / \mathrm{dL}$ and triglycerides of $145 \mathrm{mg} / \mathrm{dL}$. Microscopy showed 110 cells $/ \mathrm{mm}^{3}$, mostly lymphocytes and predominant chylomicrons. The culture of the fluid was sterile.

Ascites was slowly drained over the next 72 hours. He was put on a fat free, MCT based diet. A CT abdomen was done to look for obstruction of lymphatics, but did not reveal any abnormality. The accumulation of fluid gradually abated. In view of the same, lymphangiography or MR scanning was deferred. The subsequent course in the hospital was complicated by the occurrence of cerebral sinus thrombosis, which resolved with anticoagulant therapy and supportive care. Following discharge from the hospital, he was managed by the family doctor.
Chylous accumulation in peritoneal cavity may be caused by intestinal lymphangiectasia which may be congenital or associated with trauma, lymphoma, intestinal malignancy, pancreatitis, liver cirrhosis liver and right-sided heart failure [1]. Chylous ascites is not commonly a feature of idiopathic nephrotic syndrome in children.

A few isolated cases of chylous ascites have been described in adults with nephrotic syndrome with membranous nephropathy [2], focal segmental glomerulosclerosis [3] and renal vein thrombosis [4]. Recently chylous ascites was reported as a presenting feature in a child with systemic lupus [5]. In our patient various secondary causes of nephritic syndrome were excluded. Extensive literature search disclosed only one case of nephrotic syndrome complicated by chylous ascites in a 2-year and 8-month-old girl [6]. Repeated ascitic drainage in this girl was followed by resolution of ascites, whose proteinuria further responded to immunosuppressive drugs. The observations in this case were very similar to those being reported by us.

The mechanism of chylous ascites formation in nephrotic syndrome is not clear. It has been suggested that leakage from the dilated subserosal lymphatics from the edematous bowel mucosa and submucosa may be responsible [6]. Such lymphangiectasia may be caused by a slowing of venous return due to pressure exerted by persistent voluminous ascites. The gradual resolution of ascites with paracentesis and judicious use of diuretics supports the above hypothesis.

\section{Sonali Ghosh, ${ }^{*}$ AS VASUdeV ${ }^{2}$ and RN SRIVAStaVa ${ }^{2}$ From Departments of ${ }^{1}$ Pediatric Intensive Care and ${ }^{2}$ Pediatric Nephrology, Indraprastha Apollo hospitals, New Delhi, India. *mghosh95@gmail.com}

\section{REFERENCES}

1. Goel A, Gaur MK, Garg PK. Milk mysentery: Acute abdomen with chylous ascites. Indian Pediatr. 2018:55:909-10.

2. Kato A, Kohno S, Ohtake T, Takita T, Hirshida A.Chylous ascites in an adult patient with nephrotic syndrome due to membranous nephropathy. Nephron.2001:89:361-2.

3. Lahiri D, Agarwal R, Roy MK, Biswas A. Chylous ascites and lymphangiectasia in focal segmental glomerulosclerosis, a rare coexistence: A case report. J Med Case Rep. 2015;9:34-40.

4. LindenbaumJ,Cheidt SS.Chylous ascites and the nephrotic syndrome: Report of a case, associated with renal vein thrombosis. Am J Med. 1968:44:830-6.

5. Pilania RK, Jindal AK, Sandesh G, Vignesh P, Suri D, Nada $\mathrm{R}$,et al. Chylous ascites and podocytopathy as the presentation of childhood lupus - An unusual occurrence. Lupus. 2019;28:244-48.

6. MabialaBabela JR, Ollandzobolkobo LC, Loumingoou R, Nika ER, Mouko A. Nephrotic syndrome complicated by chylousascitis in a girl of 2 years and 8 months old. Arch Pediatr. 2017;24:24-27. 